\title{
Synthesis and Characterization of Hollow Silicon-Carbon Composites as a Lithium Battery Anode Material
}

\author{
Won-Kyu Han, Yong-Nam Ko, Chong Seung Yoon, Yong-Ho Choa*, Sung-Tag Oh** and Sung-Goon Kang ${ }^{\dagger}$ \\ Division of Materials Science and Engineering, Hanyang University, Seoul 133-791, South Korea \\ Department of Fine Chemical Engineering, Hanyang University, Ansan 426-791, Korea \\ Department of Materials Science and Engineering, Seoul National University of Technology, Seoul 139-743, Korea
}

(Received July 16, 2009 : Received in revised form September 21, 2009 : Accepted September 21, 2009)

\begin{abstract}
Si-C composite with hollow spherical structure was synthesized using ultrasonic treatment of organosilica powder formed by hydrolysis of phenyltrimethoxysilane. The prepared powder was pyrolyzed at various temperatures ranging from 900 to $1300{ }^{\circ} \mathrm{C}$ under nitrogen atmosphere to obtain optimum conditions for Li-ion battery anode materials with high capacity and cyclability. The XRD and elemental analysis results show that the pyrolyzed $\mathrm{Si} / \mathrm{C}$ composite at $1100{ }^{\circ} \mathrm{C}$ has low oxygen and nitrogen levels, which is desirable for increasing the electrochemical capacity and reducing the irreversible capacity of the first discharge. The solid Si-C composite electrode shows a first charge capacity of $\sim 500 \mathrm{mAhg}^{-1}$ and a capacity fade within 30 cycles of $0.93 \%$ per cycle. On the other hand, the electrochemical performance of the hollow Si-C composite electrode exhibits a reversible charge capacity of $\sim 540 \mathrm{mAhg}^{-1}$ with an excellent capacity retention of capacity loss $0.43 \%$ per cycle up to 30 cycles. The improved electrochemical properties are attributed to facile diffusion of Li ions into the hollow shell with nanoscale thickness. In addition, the empty core space provides a buffer zone to relieve the mechanical stresses incurred during Li insertion.
\end{abstract}

Key words hollow structure, anode, battery, Si carbon composite.

\section{Introduction}

The ubiquitous presence of portable electronic devices and development of electric vehicles have created a great demand for lithium ion batteries with increasingly high energy and power density. In Li-ion batteries, graphitic materials have been widely used as negative electrodes for lithium secondary batteries due to its low cost, high yield and long cycle life. However, the lithium storage capacity of graphite is limited to the theoretical capacity of $372 \mathrm{mAh} \mathrm{g}^{-1}$.

The demand for high energy density has stimulated the research for a new storage anode material. ${ }^{2-4)}$ Among these alternative anode materials, silicon based materials have shown a promising application in rechargeable lithiumion batteries due to its low cost and energy density. However, the cycling performance of silicon is poor, owing to its severe volume expansion and contraction during the insertion and extraction of lithium ions. The volume expansion and contraction resulted in pulverization of $\mathrm{Si}$ particles and eventual loss of $\mathrm{Li}^{+}$storage ability. To improve the capacity retention of $\mathrm{Si}$, various approaches, such as the

Corresponding author

E-Mail : sgkang@hanyang.ac.kr (S. -G. Kang) formation of mesopores ${ }^{6}$ and the use of silicon-carbon composite $^{7)}$ have been attempted. Si was also introduced in the form of nano-sized active material. ${ }^{8)}$ Recently, Li storage in hollow spheres was reported. ${ }^{9)}$ These active materials with hollow structure showed excellent rate capability and cycle retention because the hollow structure provided space to buffer the volume change in Li-alloying process and a shortened solid-state diffusion length. However, to synthesize these hollow anode materials, it is necessary to use a template such as polymer microspheres, silica spheres, and block copolymers. ${ }^{10)}$ These template-assisted synthetic methods are extremely complicated and time-consuming processes making it difficult to scale up the synthetic approach for hollow structures to an industrial level.

In this paper, we report fabrication of $\mathrm{Si}-\mathrm{C}$ hollow spheres from phenyltrimethoxysilane (PTMS) using ultrasonic treatment. A major advantage of this process is that only the sonication in ethanol is required to form a hollow structure and the size of the hollow spheres can be easily controlled by varying the immersion time. This paper examines the formation of Si-C hollow composite spheres and their electrochemical properties as an anode material for lithium-ion batteries. 


\section{Experimental Procedure}

\subsection{Synthesis of Si-C hollow composite sphere}

For the preparation of Si-C hollow composite spheres, a three-step method based on the sol-gel process was used in this study. In the first step, an aqueous solution containing $6.60 \mathrm{mmol} \mathrm{L}^{-1} \mathrm{HNO}_{3}$ was placed in an isothermal water bath at $60{ }^{\circ} \mathrm{C}$. The PTMS with $53.56 \mathrm{mmol} \mathrm{L}^{-1}$ was poured in the $\mathrm{HNO}_{3}$ solution for hydrolysis. The mixture solution was stirred at a rate of $900 \mathrm{rpm}$ for four minutes. An $\mathrm{NH}_{4} \mathrm{OH}$ solution with $1.44 \mathrm{~mol} \mathrm{~L}^{-1}$ was added to the resultant homogeneous solution for condensation. The transparent mixture solution immediately became a milky solution $\left(\mathrm{HNO}_{3}\right.$ : PTMS : $\mathrm{NH}_{4} \mathrm{OH}=1: 1.8: 218$, molar ratio). The solution was continuously aged for three hours at $60{ }^{\circ} \mathrm{C}$. The resulting powder was separated from the solution via filtering and air-dried under at room temperature. In the second step, organo-silica powder formed in the first step was poured into ethanol and then sonicated for two hours to form hollow silica spheres. In the third step, organo-hollow silica powder was pyrolyzed at various temperatures $\left(900-1500{ }^{\circ} \mathrm{C}\right)$. These samples were heated at $5{ }^{\circ} \mathrm{C} / \mathrm{min}$ and held for two hours at each temperature in a constant flow (100 ml/min) of nitrogen gas (99.99\%). In order to compare the enhanced electrochemical performance of hollow electrode, the solid electrode is also synthesized by above mentioned method without sonication (the second-step is passed).

The fabricated powder was characterized using X-ray diffractometer with $\mathrm{Cu}$ Ka radiation (XRD, Rigaku, DMax 2500). Thermogravimetric analysis (TGA, TA instruments, SDT2960) was performed at up to $1500{ }^{\circ} \mathrm{C}$ at a heating rate of $10{ }^{\circ} \mathrm{C} / \mathrm{min}$ under nitrogen atmosphere to examine the thermal behavior of the organic silica particles. The elemental analysis of the organo-silica and pyrolyzed powders was conducted to investigate the chemical composition. The morphology and microstructure of the active materials were analyzed with transmission electron microscopy (TEM, JEOL, JEM2010).

\subsection{Cell assembling and electrochemical test}

The Si-C hollow composite electrodes were prepared as followed: $80 \%$ active materials, $15 \%$ acetylene carbon black and $5 \%$ poly-vinylidene fluoride in a solvent of dimethyl phthalate were mixed. The blended slurry was then coated on a copper foil to form the electrode. After solvent evaporation, the electrode was dried under vacuum at $140{ }^{\circ} \mathrm{C}$ and finally pressed at $150 \mathrm{kgcm}^{-2}$. CR2032 coin cells were assembled in an argon-filled glove box. The counter electrode was $\mathrm{Li}$ metal and the electrolyte was $1 \mathrm{M} \mathrm{LiPF}_{6}$ dissolved in a $50: 50(\mathrm{v} / \mathrm{v})$ mixture of ethylene carbonated (EC) and dimethyl carbonate (DMC) provided by MERCK KgaA, Germany. These cells were cycled between $0.012 \mathrm{~V}$ and $1.2 \mathrm{~V}$ at room temperature.

\section{Result and discussion}

The TEM micrograph of the organo-silica powder obtained by hydrolyzing PTMS is shown in Fig. 1. Asprepared solid particles are monodispersed and in a spherical shape (Fig. 1a). The average particles size is approximately $400 \mathrm{~nm}$ in diameter. After ultrasonic treatment in ethanol, a noticeable contrast between the core and shell of particles is observed in the TEM image (Fig. 1b). The overall diameter of hollow sphere is decreased from $400 \mathrm{~nm}$ to $200 \mathrm{~nm}$ and the inner diameter of hollow
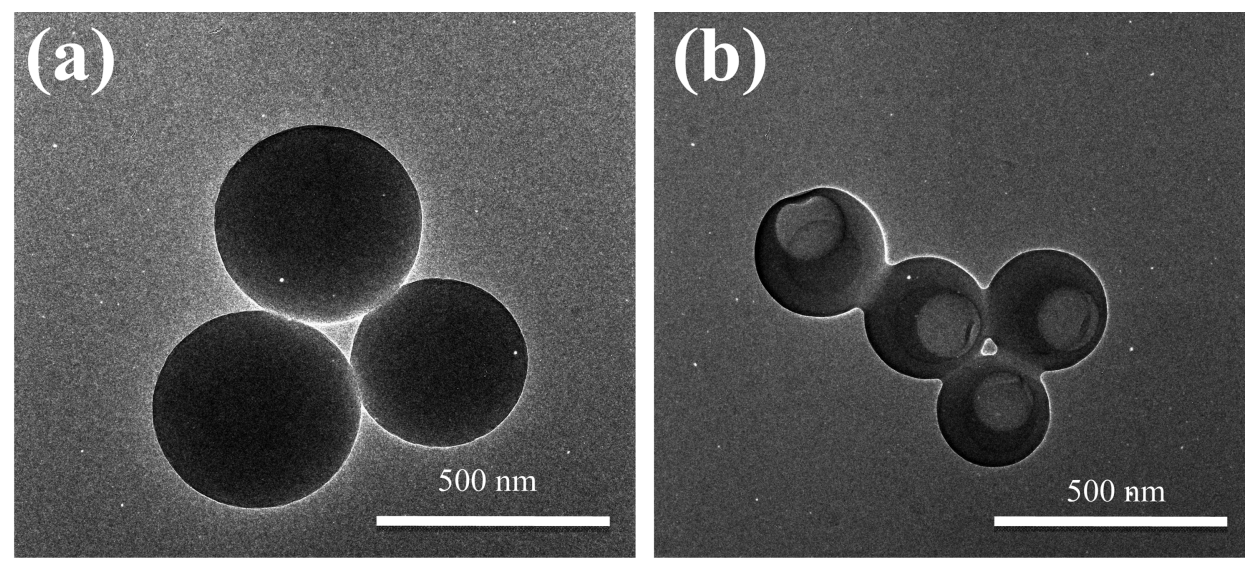

Fig. 1. SEM micrograph of (a) the as-synthesized organo-silica particles and (b) ultrasonic treated organo-silica. 

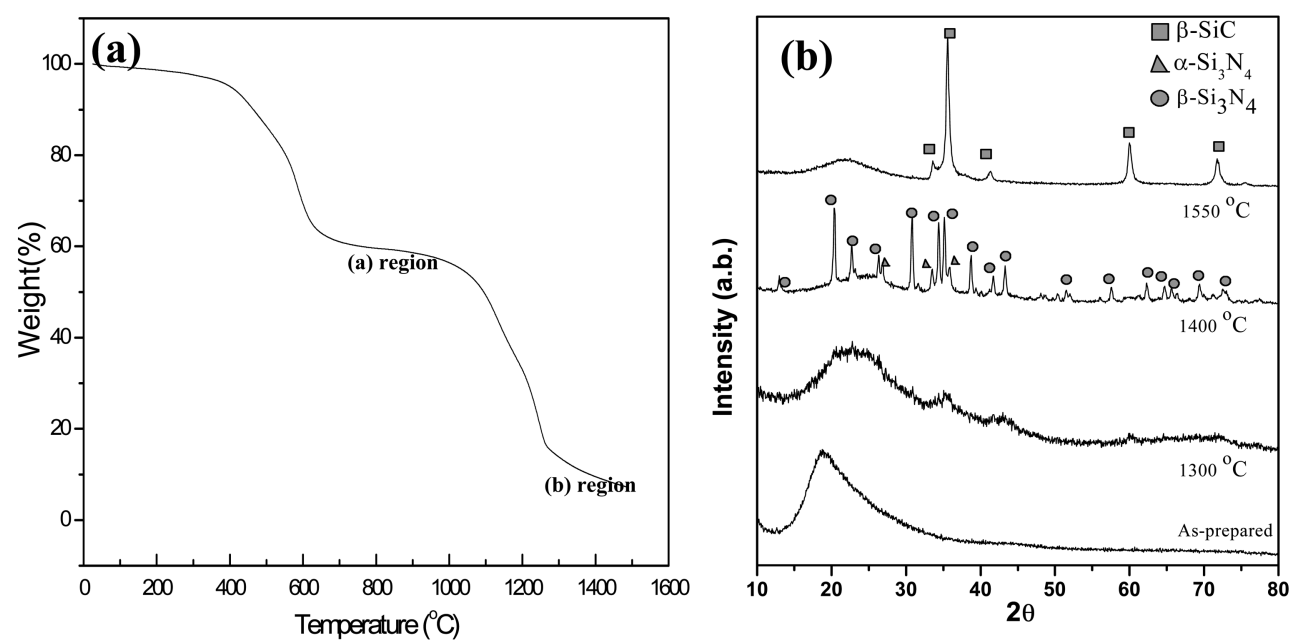

Fig. 2. (a) TGA analysis of the organo-silica powder to $1500{ }^{\circ} \mathrm{C}$ under nitrogen atmosphere. (b) XRD patterns of the organo-silica powder and powders pyrolyzed at various temperatures for $2 \mathrm{~h}$ under nitrogen.

particle is approximately $130 \mathrm{~nm}$. It clearly shows that the spheres with hollow structure are successfully formed by the proposed method. The mechanism of the formation of the hollow structure is unclear. Recently, Hah et al. insisted that the treatment in ethanol is the key procedure for the formation of a hollow structure. In other words, when as-prepared solid PTMS particles are treated in ethanol, which is permeable through the particles, linear oligomers are released from the interior of the PTMS particles during treatment. ${ }^{11)}$ As a result, the sonication of ethanol-contained PTMS particles is accelerated to the formation of hollow structure of PTMS particles.

Fig. 2a shows thermograms of the as-prepared organosilica hollow spheres under a nitrogen atmosphere, obtained by TGA. The TGA result indicates an initial weight loss of $\sim 40 \%$ at $400-600{ }^{\circ} \mathrm{C}$ and second loss that begins at approximately $1300{ }^{\circ} \mathrm{C}$. To investigate phases of the product formed by the initial and second loss reaction, organo-hollow silica powder was pyrolyzed at various temperatures for two hours and then XRD analysis was conducted (Fig. 2b). No crystalline peak is visible up to $1200{ }^{\circ} \mathrm{C}$ (data not shown). However, a small amount of $\alpha-\mathrm{Si}_{3} \mathrm{~N}_{4}$ is detected at $1300{ }^{\circ} \mathrm{C}$. When heat-treatment was performed at $1400{ }^{\circ} \mathrm{C}$, the amorphous phase crystallized to $\mathrm{Si}_{3} \mathrm{~N}_{4}$ as evidenced by well-defined sharp peaks. This phenomenon is well known that $\mathrm{Si}_{3} \mathrm{~N}_{4}$ is formed by the reaction of carbon and nitrogen gas (Eq. (1)). With increasing pyrolysis temperature of up to $1500{ }^{\circ} \mathrm{C}$, the $\mathrm{Si}_{3} \mathrm{~N}_{4}$ phase is completely transformed to $\mathrm{SiC}$ via carbothermal reduction (Eq. (2)). SiC is known to be thermodynamically
Table 1. Elemental composition of the organo-silica powder pyrolyzed at various temperatures for $2 \mathrm{~h}$ under nitrogen.

\begin{tabular}{lccccc}
\hline & \multicolumn{5}{c}{ Elemental composition (wt. \%) } \\
& Silicon & Carbon & Oxygen & Nitrogen & Hydrogen \\
\hline $900{ }^{\circ} \mathrm{C}$ & 51.58 & 37.1 & 9.83 & - & 1.49 \\
$1000{ }^{\circ} \mathrm{C}$ & 52.52 & 38.42 & 7.82 & - & 1.24 \\
$1100{ }^{\circ} \mathrm{C}$ & 55.72 & 40.32 & 2.7 & 0.51 & 0.75 \\
$1200{ }^{\circ} \mathrm{C}$ & 56.29 & 38.69 & 2.43 & 1.95 & 0.64 \\
\hline
\end{tabular}

more stable than $\mathrm{Si}_{3} \mathrm{~N}_{4}$ above a certain boundary temperature, depending on the carbon activity and the nitrogen pressure in Si-O-C-N system. ${ }^{12)}$ The reported boundary temperature is within the range of $1450-1550{ }^{\circ} \mathrm{C}^{13)}$ In our experiment, the boundary temperature shows a similar range.

$$
\begin{aligned}
& 3 \mathrm{SiO}_{2}+6 \mathrm{C}+2 \mathrm{~N}_{2} \rightarrow \mathrm{Si}_{3} \mathrm{~N}_{4}+6 \mathrm{CO} \\
& \mathrm{Si}_{3} \mathrm{~N}_{4}+3 \mathrm{C} \rightarrow 3 \mathrm{SiC}+2 \mathrm{~N}_{2}
\end{aligned}
$$

To obtain the composite with high capacity and cyclic performance, it is important to determine the pyrolysis temperature of organo-silica powder with the lower oxygen level. If the oxygen is present in the form of a Si-C-O glass, it irreversibly consumes lithium to make local arrangements like those found in $\mathrm{Li}_{2} \mathrm{O} .{ }^{14)}$ To measure the oxygen level of the composite, the element analysis was conducted ranging from 900 to $1200{ }^{\circ} \mathrm{C}$ because the composite annealed under $900{ }^{\circ} \mathrm{C}$ possesses high oxygen level contents (data not shown) and the $\alpha-\mathrm{Si}_{3} \mathrm{~N}_{4}$ is formed at over $1300{ }^{\circ} \mathrm{C}$ (as seen in Fig. 2b), which phase decreases the electrochemical activity of electrodes. As seen in Table 1, by increasing the pyrolysis temperature, the 

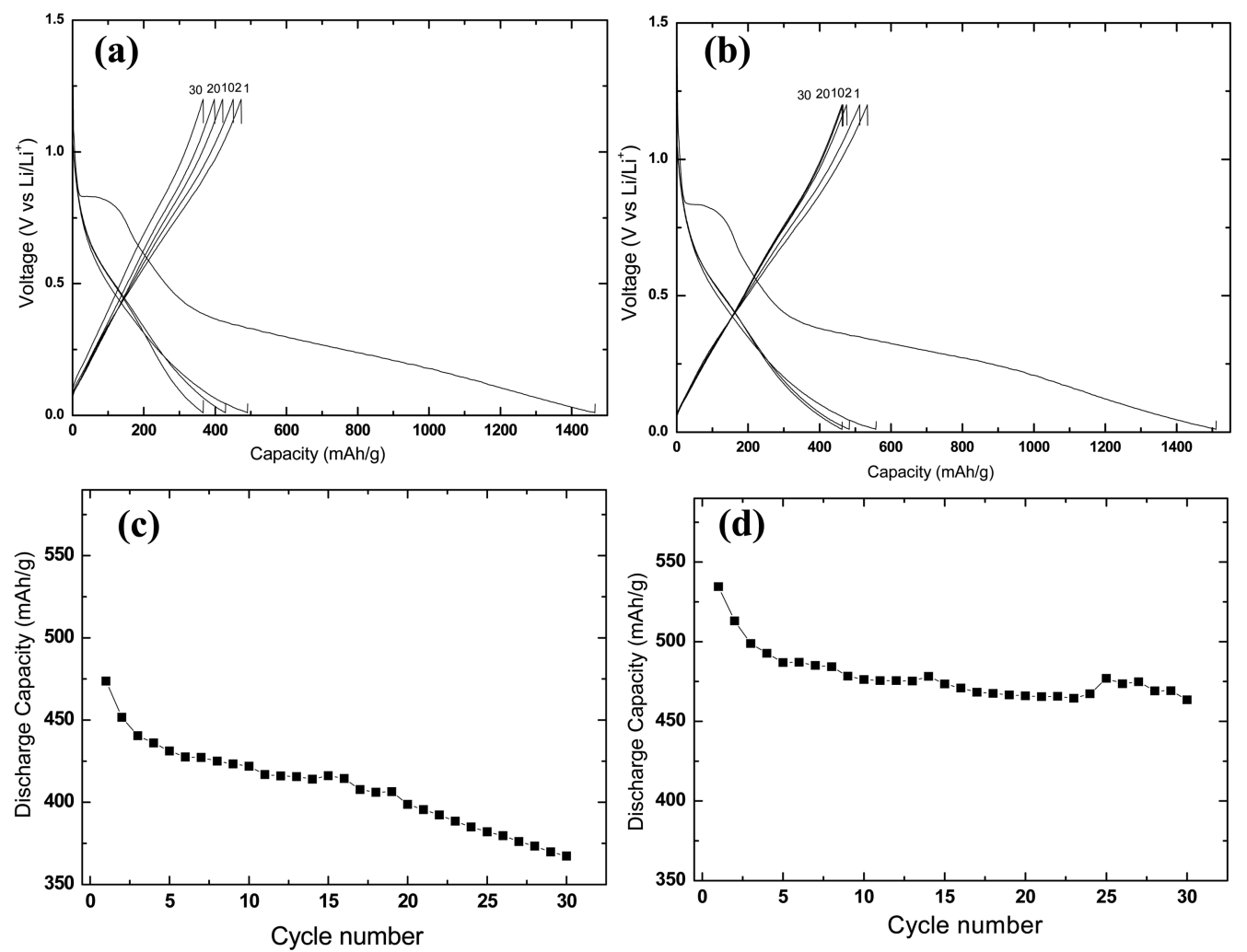

Fig. 3. Voltage profiles of the (a) solid and (b) hollow electrode after the 1st, 2nd 10th, 20th and 30th cycle at a rate of $60 \mathrm{mAg}^{-1}$ between 0 and $1.2 \mathrm{~V}$. Cycling behaviors of the (c) solid and (d) hollow electrode up to the 30 th cycle.

amount of the oxygen and hydrogen level is reduced. On the other hand, silicon content of pyrolyzed powder is steadily increased. And the carbon retains a similar value regardless of increasing the pyrolysis temperature. In the case of nitrogen, when the pyrolysis temperature is at $1100{ }^{\circ} \mathrm{C}$, the nitrogen is detected, which means that small amounts of $\mathrm{Si}_{3} \mathrm{~N}_{4}$ phase is formed in the composite (XRD does not detect small quantities $(<2 \mathrm{wt} \%))$. The above mentioned $\mathrm{Si}_{3} \mathrm{~N}_{4}$ phase can take place with a small reversible capacity of $40 \mathrm{mAhg}^{-1}$ in a potential range of 0.01-1.5 V. ${ }^{15}$ Therefore, in this study, we set the optimized pyrolysis temperature of $1100{ }^{\circ} \mathrm{C}$ for Li-ion anode materials.

Fig. 3 shows voltage and cyclic profiles of the solid and hollow structured electrodes from $1^{\text {st }}, 10^{\text {th }}, 20^{\text {th }}$ and $30^{\text {th }}$ cycles. Both electrodes were cycled between 1.2 and $0.012 \mathrm{~V}$ versus $\mathrm{Li} / \mathrm{Li}^{+}$at a current rate of $60 \mathrm{mAg}^{-1}$. As seen in Fig. 3a and c, a plateau appears at approximately $0.8 \mathrm{~V}$ in the first discharge curve of the solid electrode. The plateau may be related to the formation of a passivation film or solid electrolyte interphase (SEI) on the free carbon surface. ${ }^{16)}$ The first charge capacity is $500 \mathrm{mAhg}^{-1}$ so that only $66 \%$ of the first discharge capacity is re- covered during the first charge cycle. The large irreversible capacity loss in the first cycle is likely due to the formation of SEI. ${ }^{17)}$ The capacity of the solid electrode after 30 cycles is $360 \mathrm{mAhg}^{-1}$. On the other hand, as seen in Fig. $3 b$ and $d$, the voltage profile of hollow electrode during the first discharge and charge has a similar but an observation of the more SEI reaction than solid electrode, which is attributed to the large surface area of hollow electrode. The electrode with hollow structure showed first discharge and charge capacities of $1520 \mathrm{mAhg}^{-}{ }^{1}$ and $540 \mathrm{mAhg}^{1}{ }^{1}$, respectively and its capacity after 30 cycles is $470 \mathrm{mAhg}^{-1}$. The capacity retention after 30 cycles was $87 \%$ of the first-charge capacity for the hallow electrode. This value is far superior to that of the solid electrode. The improved capacity and cycle retention of the hallow electrode are related to the hollow structure of the anode, which provides a large surface area and a shorter solidstate diffusion length. ${ }^{18)}$ In addition, the empty core structure can act as a buffer space to accommodate the stress arising from the volume change of the PTMS shell during Li-insertion. 


\section{Conclusion}

In this paper, the PTMS electrode with hollow structure for Li-ion batteries was successfully fabricated by ultrasonication in ethanol. The synthesized hollow electrode exhibits improved electrochemical properties such as cycle capacity and capacity retention, compared to the solid PTMS electrode when cycled at a rate of $60 \mathrm{mAg}^{-1}$. We believe that the enhanced cycle performance is due to the empty core of the hollow electrode, acting as a buffer zone which accommodates the volume change of the Si phase during the $\mathrm{Li}$ intercalation/de-intercalation. The hollow structure also provides a large surface area and shorter solid-state diffusion length, leading to higher energy density than the solid counterpart.

\section{Acknowledgement}

This work was supported by the Korea Science and Engineering Foundation (KOSEF) (NO. R01-2007-000-20703-0).

\section{References}

1. B. A. Johnson and R. E. White, J. Power Sources, 70, 48 (1998).

2. J. Yang, M. Winter and J. O. Besenhard, Solid State Ionics, 90, 281 (1996).
3. J. O. Besenhard, J. Yang and M. Winter, J. Power Sources, 68, 87 (1997).

4. W. K. Han, Y. H. Choa, S. T. Cho and S. G. Kang, Kor. J. Mater. Res., 18(4), 187 (2008).

5. R. A. Sharma and R. N. Seefurth, J. Electrochem. Soc., 123, 1763 (1976).

6. Y. Zheng, J. Yang, J. Wang and Y. NuLi, Electrochim. Acta, 52, 5863 (2007).

7. K. Hanai, Y. Liu, N. Imanishi, A. Hirano, M. Matsumura, T. Ichikawa and Y. Takeda, J. Power Sources, 146, 156 (2005).

8. H. Li, X. Huang, L. Chen, Z. Wu and Y. Liang, Electrochem. Solid-State Lett., 7, 547 (1997).

9. Y. Wang, F. Su, J. Y. Lee and X. S. Zhao, Chem. Mater., 18, 1347 (2006).

10. S. G. Han, B. C. Jang, T. A. Kim, S. M. Oh and T. H. Hyeon, Adv. Funct. Mater., 15, 1845 (2005).

11. H. J. Hah, J. S. Kim, B. J. Jeon, S. M. Koo and Y. E. Lee, Chem. Commun., 14, 1712 (2003).

12. M. J. Wang and H. Wada, J. Mater. Sci., 25, 1690 (1990).

13. H. Wang and G. S. Fischman, J. Am. Ceram. Soc., 74(1), 1519 (1991).

14. A. M. Wilson, G. Zank, K. Egushi, W. Xing and J. R. Dahn, J. Power Sources, 68, 195 (1997).

15. M. Martin-Gil, M. E. Rabanal, A. Varez, A. Kuhn and F. Garcia-Alvarado, Mater. Lett., 57, 3063 (2003).

16. M. H. Chen, Z. C. Huang, G. T. Wu, G. M. Zhu, J. K. You and Z. G. Lin, Mater. Res. Bull., 38, 831 (2003).

17. Y. Wang and J. Y. Lee, J. Power Sources, 144, 220 (2005).

18. K. T. Lee, J. C. Lytle, N. S. Ergang, S. M. Oh and A. Stein, Adv. Funct. Mater., 15, 547 (2005). 